\title{
Ter- dragon curve: a view in cordial and edge cordial labeling
}

\author{
A.A. Sathakathulla \\ University of Modern Sciences, Dubai, U.A.E \\ E-mail: aasathak@gmail.com
}

Copyright $\odot 2014$ A.A. Sathakathulla. This is an open access article distributed under the Creative Commons Attribution License, which permits unrestricted use, distribution, and reproduction in any medium, provided the original work is properly cited.

\begin{abstract}
A fractal is a mathematical set that typically displays self-similar patterns. The Ter dragon curve is also a fractal in the family of $\sqrt{ } 3$ curve in brain filling curve models. There are many in this family of curves but for my study I have considered this fractal curve. This fractal has been considered as a graph and the same has been viewed under the cordial and edge cordial labeling to apply this curve with scope for further study.
\end{abstract}

Keywords: Ter-Dragon Curve, Brain Filling Fractal, Cordial, Edge Cordial, Graph.

\section{Introduction}

The root 3 family of curves is one of the families of plane- filling curves. Unlike the fractal curves of the root 2 family, these curves live in the triangular grid. It has a fractal generator in the triangular grid. It has been found that there are ten plane-filling curves of the root 3 families. One of them is familiar to fractal as Ter-dragon. Famed Computer scientist Donald Knuth discovered the Ter-dragon. The beauty of Ter-dragon is, it has point symmetry and its tail looks like its head and which looks like it lies in its tail. Further, it has been noticed that three copies of the Ter-dragon can be combined to make a larger one.

The Ter-dragon is the first example of palindrome Curve, that is, a fractal curve which is symmetrical about its center. Palindrome Dragons have heads that look like upside-down copies of their tails. Based on the Ter Dragon's generator, we can create an entirely different palindrome curve simply by flipping each of the segments.

Many of its properties were published and discussed more by many scholars. In this paper, our study is to enable the Cordial, Total cordial, Edge cordial and total edge cordial labeling for the above mentioned Ter-dragon curve. So In this study, the Ter-dragon curve has been considered as a Graph with number of vertices and edges. The construction of the graph is also part of the study of properties of the curve.

Furthermore, for our study, the following definitions are to be reintroduced for clear understanding of this paper.

A Graph $\mathrm{G}=\langle\mathrm{V}, \mathrm{E}, \psi\rangle$ consists of a non-empty set $\mathrm{V}$ called the set of nodes (points, vertices) of the graph, E is said to be the set of edges (may be empty) of the graph and $\psi$ is the mapping from the set of edges $\mathrm{E}$ to a set of ordered or unordered pair of elements of V. It would be convenient to write a graph Gas <V, E > or simply as G.

A graph labeling is an assignment of integers to the vertices or edges, or both subject to certain conditions. Many types of labeling like harmonious, graceful, etc. are used by various researchers [3], [4], [6] in practice. A graph $\mathrm{G}$ with $\mathrm{q}$ edges is harmonious if there is an injection $\mathrm{f}$ from the vertices of $\mathrm{G}$ to the group of integers modulo $\mathrm{q}$ such that when each edge ' $x y$ ' is assigned the label $|f(x)+f(y)|(\bmod q)$, the resulting edge labels are distinct

A graph $\mathrm{G}$ with $\mathrm{q}$ edges is graceful if $\mathrm{f}$ is an injection from the vertices of $\mathrm{G}$ to the set $\mathrm{f}: \mathrm{V} \rightarrow\{0,1 \ldots \mathrm{q}\}$ such that, when each edge 'xy' is assigned the label $|f(x)-f(y)|$, the resulting edge labels are distinct. Eventually, after the introduction of the concept of cordial labeling by (I. Cahit, [2]) many researchers have investigated graph families or graphs which admit cordial labeling with minor variations in cordial theme like product cordial labeling, total product cordial labeling and prime cordial labeling (F. Harary [4]). The brief summary of definitions which is useful for the present investigations is given below.

Definition 1.1: if the vertices of the graph are assigned values subject to certain conditions, then it is known as graph labeling. 
For a dynamic survey on graph labeling we refer to (J.A. Gallian, [3]). A detailed study on variety of applications of graph labeling is reported in (G. S. Bloom, [1]).

Definition 1.2: Let $\mathrm{G}$ be a graph. A mapping $\mathrm{f}: \mathrm{E}(\mathrm{G}) \rightarrow\{0,1\}$ is called binary edge labeling of $\mathrm{G}$ and $\mathrm{f}(\mathrm{e})$ is called the label of the edge e of $G$ under $f$.

For an edge $\mathrm{e}=\mathrm{uv}$, the induced edge labeling $\mathrm{f}^{*}: \mathrm{E}(\mathrm{G}) \rightarrow\{0,1\}$ is given by $\mathrm{f}^{*}(\mathrm{e})=|\mathrm{f}(\mathrm{u})-\mathrm{f}(\mathrm{v})|$. Let $\mathrm{vf}(0)$, vf $(1)$ be the number of vertices of $G$ having labels 0 and 1 respectively under $f$ while ef $(0)$, ef (1) be the number of edges having labels 0 and 1 respectively under $\mathrm{f}^{*}$.

Definition 1.3: A binary vertex labeling of a graph $G$ is called a cordial labeling if $|v f(0)-v f(1)| \leq 1$ and $|e f(0)-e f(1)| \leq 1$. A graph $G$ is cordial if it admits cordial labeling.

Definition 1.4: Let $G$ be a graph with two or more vertices then the total graph $T(G)$ of a graph $G$ is the graph whose vertex set is $\mathrm{V}(\mathrm{G}) \cup \mathrm{E}(\mathrm{G})$ and two vertices are adjacent whenever they are either adjacent or incident in $\mathrm{G}$.

Definition 1.5: A binary edge labeling of a graph $G$ is called an edge cordial labeling if $|v f(0)-v f(1)| \leq 1$ and $|e f(0)-e f(1)| \leq 1$. A graph $G$ is edge cordial if it admits cordial labeling

Definition 1.6: Cahit [2] introduced edge-cordial labeling as a binary edge labelingf: $E(G) \rightarrow\{0,1\}$, with the induced vertex labeling given by $f(v)=\sum_{u v \in E} f(u v)(\bmod 2)$ for each $v \in V$ such that $\mid$ ef $(0)-$ ef (1) $\leq 1$. And $|v f(0)-v f(1)| \leq 1$, where ef(i) and vf(i) $(\mathrm{i}=0,1)$ denote the number of edges and vertices labeled with 0 and 1 respectively.

Definition 1.7: As an extension of the above, we define a total edge-cordial labeling of a graph $\mathrm{G}$ with vertex set $\mathrm{V}$ and edge set $\mathrm{E}$ as an edge-cordial labeling such that number of vertices and edges labeled with 0 and the number of vertices and edges labeled with 1 differ by at most 1 (i.e) $\left|\left(v_{f}(0)+e_{f}(0)\right)-\left(v_{f}(1)+e_{f}(1)\right)\right| \leq 1$. A graph with a total edgecordial labeling is called a total edge-cordial graph.

The present work is focused on cordial and edge cordial labeling of Ter-dragon curve at every iteration.

\section{Main results}

The construction and its properties are already well investigated by many scholars. Here, we provide the Pattern of the dragon diagram for the need of further study.
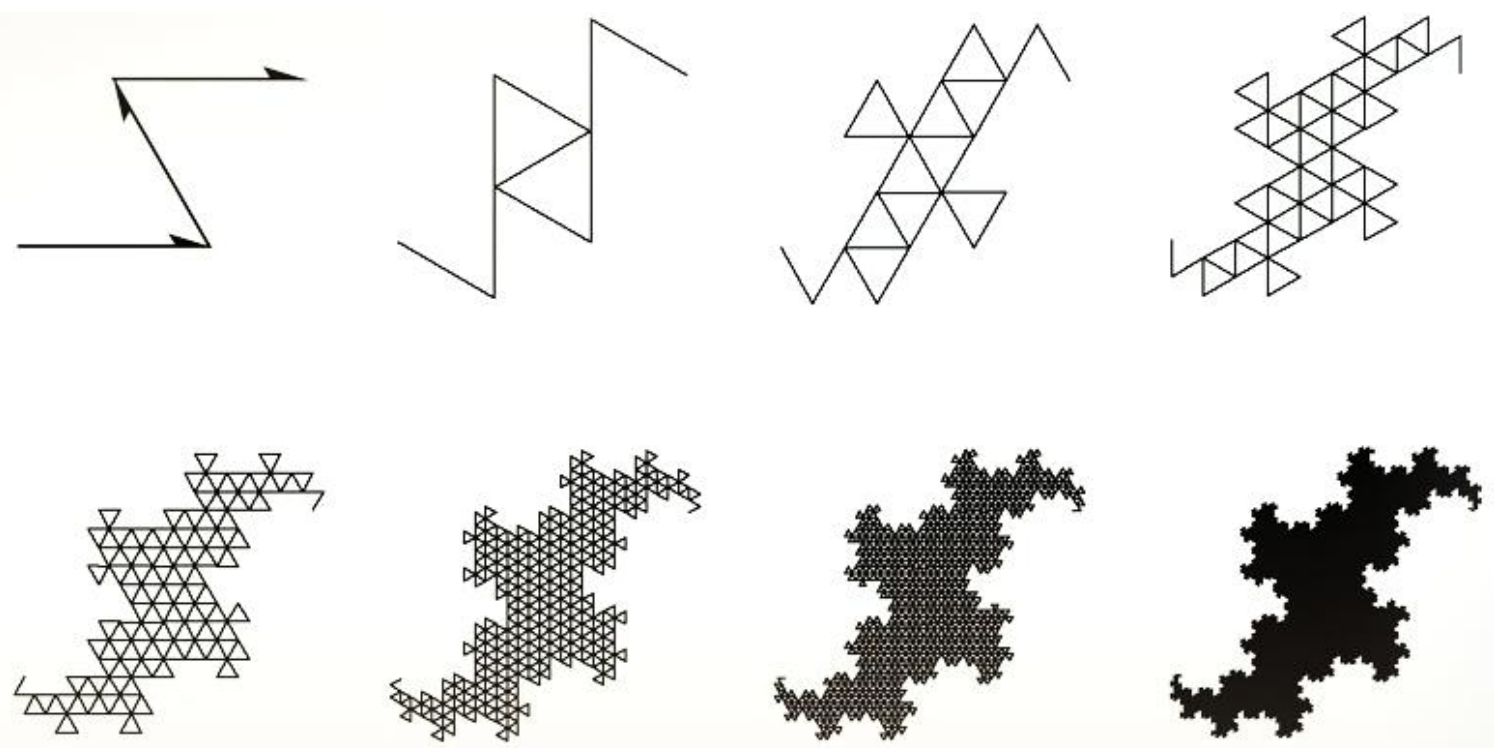

Fig. 2.1: Construction of Highway Dragon Fractal

\subsection{Cordial labeling for Ter-dragon curve}

The pattern of labeling of vertices is easily understandable by seeing the figure (Fig 2.2). The Ter dragon curve joins and intersects in each iteration developing further. The vertices are numbered 0's and 1's to satisfy the condition of cordial and total cordial in each iteration. It is clearly observed that all the edges having label 0's are denoted by a tick mark $(\sqrt{ })$ and correspondingly all edges with 1 's are denoted by a cross mark(x). Hence it satisfies the existence of 
cordial labeling. In each iteration, the similar fashion of labeling is being tried to extend and is followed to check the existence of cordial labeling and total cordial labeling. Hence, the above fractal holds good for both cordial and total cordial labeling. The iteration wise no of edges and vertices are provided in the table 2.1. Every iteration values hold good for the mentioned definitions.
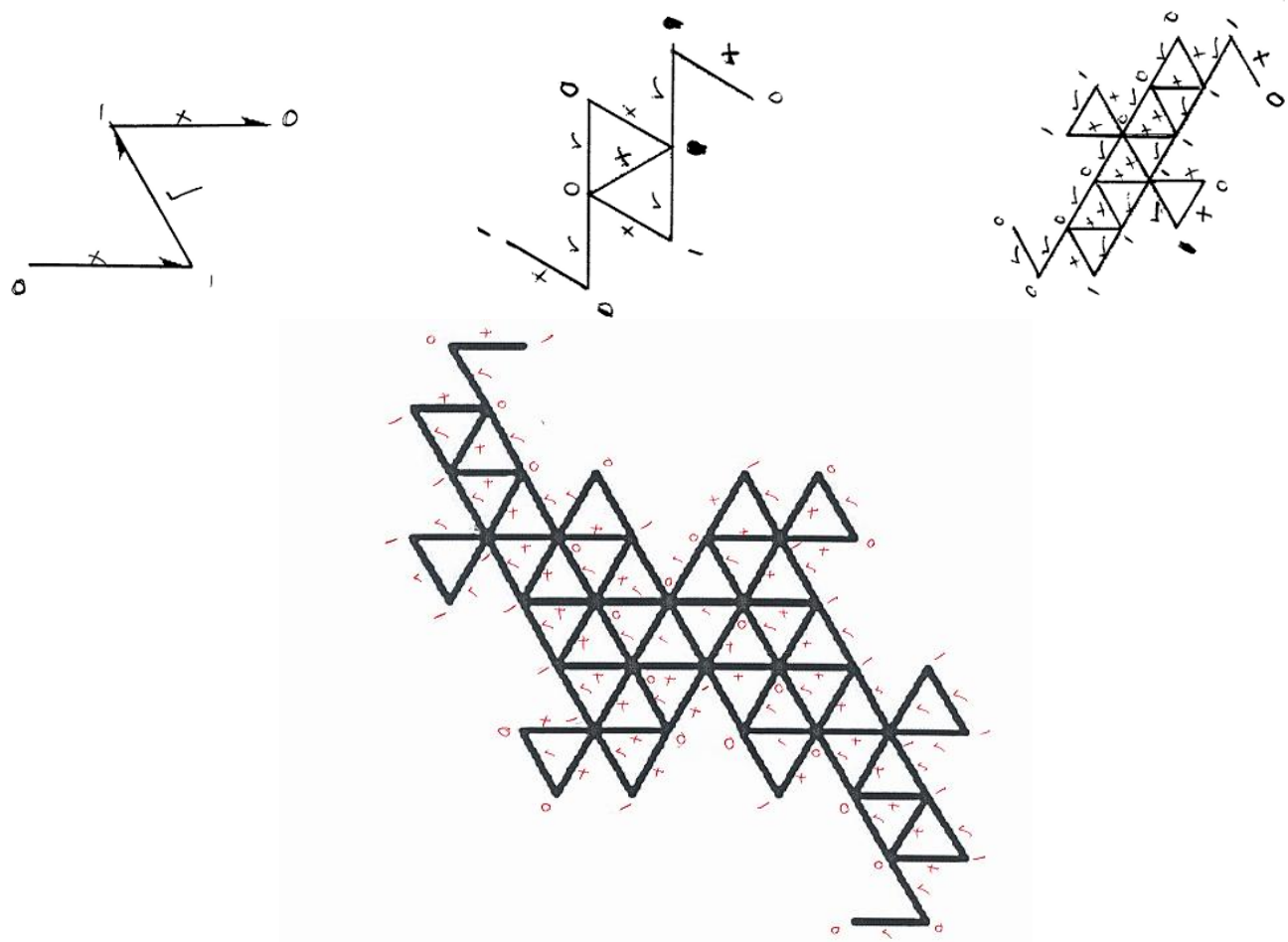

Fig. 2.2: Cordial Labeling For First Few Iterations.

\subsection{Edge cordial labeling for Ter-dragon curve}

Similar to the above, the pattern of labeling of edges is easily understandable by seeing the below figure (Fig 2.3). This fractal graph is labeled with zeros and ones at the edges by a tick mark $(\sqrt{ })$, a cross mark $(\mathrm{x})$ at vertices to distinguish. Furthermore, the same pattern has been extended to every iteration to fulfill the definition. The definition of edge cordial labeling satisfies at every iteration. In addition to that, it satisfies Total edge cordial conditions also. The results provided in the following table hold well for all iterations.
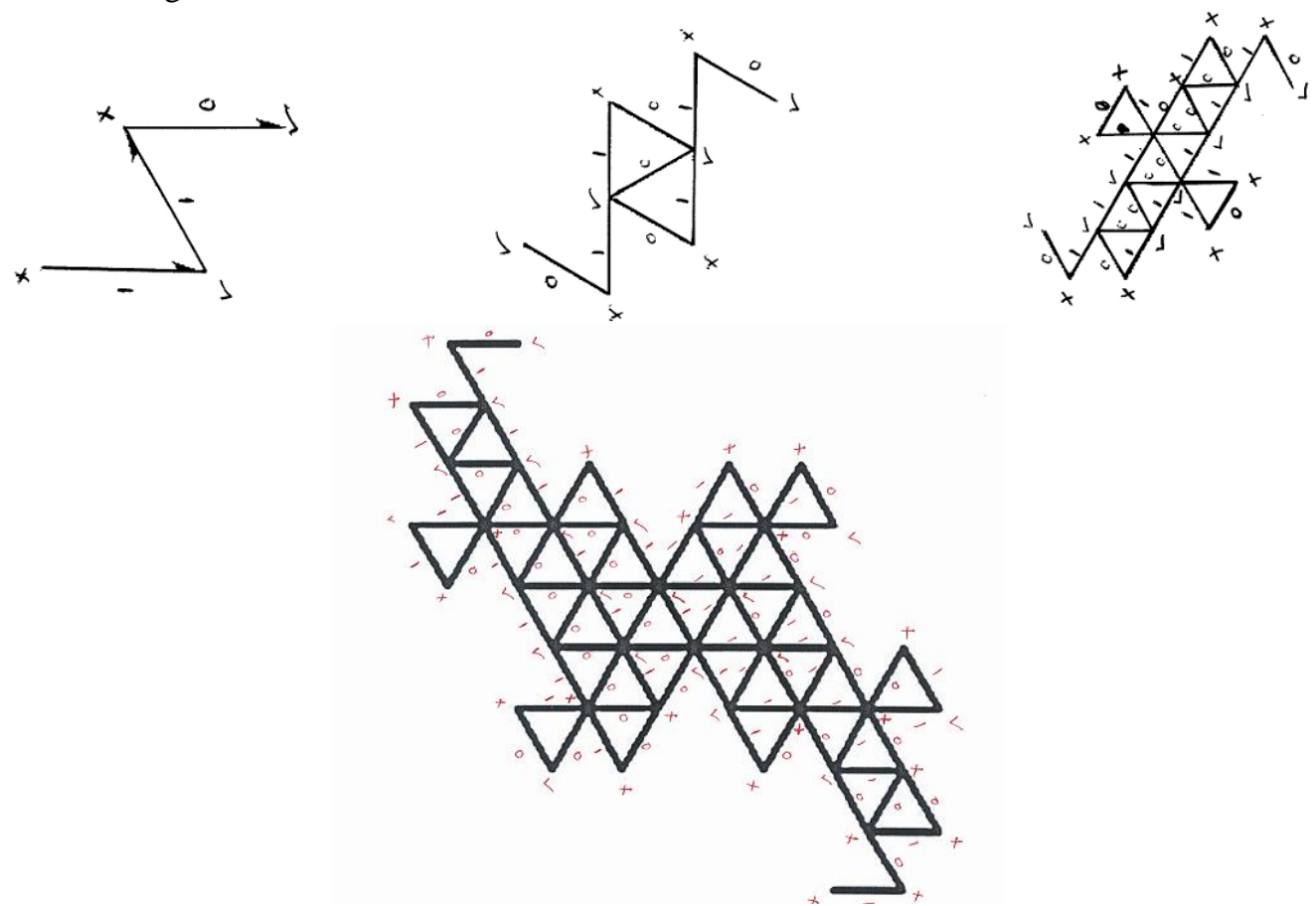

Fig. 2.3: Edge Cordial Labeling For First Few Iterations. 
The following table gives the detailed results for individual iteration wise number of edges and vertices and the results hold good for both theories of Cordial and edge cordial labeling. Moreover, this table proves that the results hold good for Total cordial and total edge cordial labeling too. Since there are more intersecting vertices, by using the induction method, the number of vertices and corresponding number of 0 's and 1 's is calculated for further iterations.

Table 2.1: Number of vertices and edges

\begin{tabular}{|c|c|c|}
\hline Iterations & No. of Vertices & No. of Edges \\
\hline 1 & $|\mathrm{v}|=4 \quad$ and $|\mathrm{vf}(0)|=|\mathrm{vf}(1)|=2$ & $|\mathrm{e}|=3$ and $|\mathrm{ef}(0)|=1 \quad|\mathrm{ef}(1)|=2$ \\
\hline 2 & $|\mathrm{v}|=8 \quad$ and $\quad|\operatorname{vf}(0)|=|\operatorname{vf}(1)|=4$ & $|\mathrm{e}|=3^{2}$ and $|\mathrm{ef}(0)|=4 \quad \mid \mathrm{ef}(1)=5$ \\
\hline 3 & $|\mathrm{v}|=18$ and $|\operatorname{vf}(0)|=|\mathrm{vf}(1)|=9$ & $|\mathrm{e}|=3^{3}$ and $|\mathrm{ef}(0)|=14 \quad$ ef $(1) \mid=15$ \\
\hline 4 & $|\mathrm{v}|=44$ and $|\mathrm{vf}(0)|=|\mathrm{vf}(1)|=22$ & $|\mathrm{e}|=3^{4}$ and $|\mathrm{ef}(0)|=40 \quad|\mathrm{ef}(1)|=41$ \\
\hline 5 & $|\mathrm{v}|=114$ and $|\mathrm{vf}(0)|=|\mathrm{vf}(1)|=57$ & $|\mathrm{e}|=3^{5}$ and $|\mathrm{ef}(0)|=121|\mathrm{ef}(1)|=122$ \\
\hline 6 & $|\mathrm{v}|=308$ and $|\mathrm{vf}(0)|=|\mathrm{vf}(1)|=154$ & $|\mathrm{e}|=3^{6}$ and $|\mathrm{ef}(0)|=364|\mathrm{ef}(1)|=365$ \\
\hline $\mathrm{n}$ & $\begin{array}{l}\text { If } n \text { is Even } \\
|v|=44 \times 7^{\lfloor n / 2\rfloor-2} \quad \text { for } n \geq 4 \text { If } n \text { is odd } \\
|v|=16 \times 7^{\lfloor n / 2\rfloor-1}+2 \quad \text { for } n \geq 3 \\
\text { and }|v f(0)|=|v f(1)|=|v| / 2\end{array}$ & $\begin{aligned}|e|=3^{n} \text { and } & |e f(0)|=\left\lfloor 3^{n} / 2\right\rfloor \\
& |e f(1)|=\left\lfloor 3^{n} / 2\right\rfloor+1\end{aligned}$ \\
\hline
\end{tabular}

\section{Conclusion}

In this paper, Ter-dragon has been viewed in the form of a fractal graph with the concept of labeling particularly, cordial, edge cordial labeling and total cordial, total edge cordial concepts. It is believed that this view of labeling will lead to further study of this Ter-dragon fractal graph in to other types of labeling in graph and emerging application of this Ter-dragon curve in the field of Engineering and science. The existence of above mentioned labeling is proved and the results are provided as detailed in the above table. Hence, it is concluded that the Ter-Dragon curve fractal graph is cordial, total cordial, edge cordial and total edge cordial.

\section{References}

[1] Bloom G. S. and Golomb S. W. (1977). Applications of numbered undirected graphs, Proc of IEEE, 65(4), 562-570. http://dx.doi.org/10.1109/PROC.1977.10517.

[2] Cahit I. (1987). Cordial Graphs: A weaker version of graceful and harmonious Graphs, Ars Combinatoria, 23,201-207.

[3] Gallian, J. A. (2009). A dynamic survey of graph labeling, The Electronic Journal of Combinatorics, 16 , \#DS 6.

[4] Harary, F. (1972). Graph Theory, Massachusetts, Addison Wesley.

[5] James Gleick, Chaos, Vintage Publishers, 1998.

[6] M. Barnsley, Fractals Everywhere, Academic Press Inc., 1988

[7] M. Seoud and A. E. I. Abdel Maqsoud, "On cordial and balanced labelings of graphs", Journal of Egyptian Math. Soc., Vol. 7, pp. 127-135, 1999.

[8] R. Devaney and L. Keen, eds., Chaos and Fractals: The Mathematics behind the Computer Graphics, American Mathematical Society, Providence, RI, 1989

[9] Sundaram M., Ponraj R. and Somasundram S. (2005). Prime Cordial Labeling of graphs, J.Indian Acad. Math., 27(2), 373-390

[10] Sathakathulla A.A., Muhammad Akram, (2012) A Note on Cordial, Edge Cordial Labeling of Pythagoras Tree Fractal Graphs International Journal of Scientific \& Engineering Research Volume 3, Issue 12, December-2012 